\title{
Fabienne Duteil-Ogata, Isabelle Jonveaux, Liliane Kuczynski, Sophie Nizard (dir.), Le religieux sur Internet
}

Paris, L'Harmattan, coll. « Religions en questions », AFSR, 2015, 324 p.

Charlotte Blanc

\section{OpenEdition}

Journals

Édition électronique

URL : http://journals.openedition.org/assr/28230

DOI : $10.4000 /$ assr.28230

ISSN : $1777-5825$

Éditeur

Éditions de l'EHESS

Édition imprimée

Date de publication : 31 décembre 2016

Pagination : 305

ISSN : 0335-5985

Référence électronique

Charlotte Blanc, « Fabienne Duteil-Ogata, Isabelle Jonveaux, Liliane Kuczynski, Sophie Nizard (dir.), Le religieux sur Internet », Archives de sciences sociales des religions [En ligne], 176 | octobre-décembre 2016, mis en ligne le 17 juillet 2017, consulté le 24 septembre 2020. URL : http:// journals.openedition.org/assr/28230 ; DOI : https://doi.org/10.4000/assr.28230

Ce document a été généré automatiquement le 24 septembre 2020.

(c) Archives de sciences sociales des religions 


\title{
Fabienne Duteil-Ogata, Isabelle Jonveaux, Liliane Kuczynski, Sophie Nizard (dir.), Le religieux sur Internet
}

Paris, L'Harmattan, coll. « Religions en questions », AFSR, 2015, 324 p.

\author{
Charlotte Blanc
}

\section{RÉFÉRENCE}

Fabienne Duteil-ogata, Isabelle Jonveaux, Liliane Kuczynski, Sophie Nizard (dir.), Le religieux sur Internet, Paris, L'Harmattan, coll. « Religions en questions », AFSR, 2015, $324 \mathrm{p}$.

1 Cet ouvrage, composé de contributions de vingt chercheurs et chercheuses s'intéressant aux relations entre faits religieux et Internet, fait suite au colloque organisé à Paris en février 2013, Le religieux sur Internet/Religion on the Web, ayant donné lieu à une publication dans le Online Heidelberg of religions on the Internet (vol. 8, 2015) où l'on retrouve sept des articles de l'ouvrage traduits en langue anglaise.

2 Le religieux sur Internet comporte quatre parties thématiques et problématiques: « Redessiner le paysage religieux », "Modalités et enjeux de la présence en ligne», «Pratiques et rituels en ligne», "Questions d'autorité». Formant un tout pluridisciplinaire, les contributions sont toutefois à dominante sociologique, anthropologique et communicationnelle. Si les questions religieuses intéressent le monde universitaire, les chercheurs français ont tardé à porter attention aux manifestations numériques du religieux. Cette entreprise scientifique est de ce fait à saluer tant les études francophones manquent encore de dynamisme. Ce champ de recherche déjà développé outre-Altlantique a donné un cadre théorique : les notions de online religion et religion online (Helland, 2000) structurent notamment les recherches 
dans le domaine. Notons que ce qui relève en grande partie du champ de recherche "Religion et Média» aux États-Unis et au Canada, dont les sciences de la communication se sont emparées, intéresse davantage les sociologues et anthropologues en France. Comme le soulignent les directrices de l'ouvrage, un équivalent français existe pourtant à travers, notamment, les travaux du réseau relicom (Douyère, Dufour et Riondet, MEI, n 38, 2014 ; Douyère, tic\&societé, vol. 9, 2016 - non paru à la sortie de l'ouvrage) questionnant les rapports entre communication et religion.

3 En prenant appui sur les travaux d'Antonio Casilli (2010), les directrices de l'ouvrage placent ce dernier dans le champ des cultures numériques. Elles entendent faire du religieux une " porte d'entrée des études sur Internet », (p.13), le fait religieux étant un oublié des études sur les cultures numériques et ce malgré la production de discours utopiques/dystopiques sur la nature d'Internet. Les contributions de l'ouvrage ont toutes le souci de proposer une réflexion et une analyse des « rapports entre Internet et religions en les replaçant dans des contextes culturels précis»(p.17). Les articles témoignent d'un ancrage dans le terrain dit "réel», un terrain "au sens [plus] classique du terme » (p. 21). "Réel», car l'ensemble s'applique à analyser les relations entre le fait religieux (dans toute sa variété) et le média, en essayant de saisir les mutations ou hybridités qu'Internet peut produire sur le religieux, dans ses dimensions identitaire, organisationnelle ou rituelle. Aborder Internet comme ayant un " impact » sur les pratiques religieuses a cependant pour effet de ne pas le considérer comme terrain à part entière. Ainsi, une large part des contributions considère le " hors-ligne " comme terrain de recherche principal et le « en ligne» comme l'extension de celui-ci. Des contributions telles que celles de Mira Niculescu, Pierre-Yves Kirschleger, Pascal Lardellier, et Mia Lövheim, abordent Internet non pas comme extension médiatique de pratiques « réelles ", mais comme terrain prioritaire. D'autres développent une analyse multisituée, à cheval entre les productions hors-ligne et en-ligne, comme David Douyère et Damien Mottier.

4 La première partie de l'ouvrage s'emploie à discuter et analyser d'éventuelles recompositions du "paysage religieux». Cette expression semble recouvrir les potentielles mutations des pratiques religieuses dans leur ensemble, en même temps qu'elle entend questionner la distinction entre « réel » et « virtuel ». Dans « Dieu, la noosphère et Internet ", article initialement en anglais, Olivier Krüger travaillant sur la réception culturelle des médias, questionne le cyberespace comme idéal communautaire à travers l'opérativité des notions de noosphère et de village global et cherche à comprendre le rôle d'Internet dans l'émergence d'une simultanéité entre nouveaux moyens de communication et utopies communautaires, qu'elles soient séculières ou religieuses. Selami Varlik, dans «Le discours islamique sur Internet: oralité de l'écriture et fragmentation de l'autorité ", met en avant la différenciation entre l'écriture produite hors-ligne et celle présente en ligne. Internet favoriserait une mise en récit plurielle de l'Islam et donc un processus interprétatif fondé sur un "discours participatif» (p. 54) et une «fragmentation du contenu» (p. 56). Dans «La virtualité comme catégorie religieuse?", Isabelle Jonveaux, questionne la pertinence des catégories réel/virtuel en se fondant sur une étude de cas. Elle s'intéresse à l'efficacité symbolique des pratiques en ligne à travers une étude des monastères catholiques horsligne/en ligne. Lionel Obadia interroge lui aussi la pertinence de ces catégories, dans "Quand la virtualité façonne la réalité sociale : les fake cults et la Church of the Flying Spaghetti Monster». En s'appuyant sur la production indigène du numérique d'une 
religion à caractère parodique, il montre qu'Internet et ses technologies de la communication peuvent faire émerger une continuité dans des réalités matérielles. Dans " Les relations entre religion en ligne et hors ligne dans une société en réseaux " déjà paru en anglais dans le Journal of the American Academy of Religion en 2012, Heidi Campbell, spécialiste des liens entre médias et religions dans une approche communicationnelle, met en avant un outil interprétatif des évolutions du religieux sur le web et, par extension, hors web, à travers le concept de "religion en réseau ». Sur la base d'un état de l'art des différentes problématiques ayant animé les chercheurs sur Internet et religion, et dans une démarche holiste, Campbell propose aussi une posture, celle de saisir les mutations du fait religieux à partir des deux terrains (matériel et numérique). L'ouvrage collectif questionne les différentes dimensions du concept de «religion en réseau » mises en avant par Campbell, à savoir l'identité narrative, les mutations de l'autorité, la réalité multisituée, la convergence des pratiques et la communauté en réseaux.

5 La deuxième partie s'engage à présenter différentes modalités de présence en ligne de groupes religieux divers (anglicans, protestants, juifs-bouddhistes) et à questionner les enjeux de cette présence sur les réalités matérielles. Dans «La mission d'internationaliser Saint Nicolas", André Julliard, à partir de son terrain - la spiritualité nicolaïenne, processions et autres dévotions - observe les utilisations d'un site internet dédié à Saint Nicolas (stnicholascenter.org) - pour en isoler les ruptures et continuités avec son terrain. Interrogeant d'autres modalités de présence en ligne, Mira Niculescu, s'intéresse dans « Se connecter et prendre l'avion », à la production indigène du web d'une spiritualité et d'une pratique hybrides: la Jewish Mindfulness, composite américain d'un judaïsme inspiré par les pratiques de méditation bouddhiste, et observe le devenir matériel d'une organisation initialement en ligne. Elle met ainsi en avant l'importance du « rôle de la communication en ligne dans la fabrique du religieux horsligne» (p.143). Cette partie permet, entre autres, de prendre conscience d'une implication différenciée du religieux sur le web en fonction des appartenances confessionnelles. Ainsi, Kirschleger, dans "Aux commandes du premier réseau protestant francophone: Jesus.net", s'intéresse aux usages des technologies de l'information et de la communication dans la diffusion de la foi, mais surtout dans la multiplication des pratiques religieuses en ligne et de l'enseignement religieux, qui sont autant d'indices d'émergence d'une « cyber-religion ». Ce que relevait Niculescu, le rôle du médium Internet dans l'élaboration et dans l'évolution du religieux hors-ligne, est présenté par Kirschleger de façon plus accentué, par le poids considérable joué par un usage multisitué des nouvelles technologies chez les pentecôtistes. Damien Mottier, dans "Le télé-fidèle existe-t-il ?", montre à travers une enquête de terrain sur les pratiques technologiques et numériques d'une paroisse pentecôtiste, que le numérique est plus qu'un support à l'évangélisation, mais qu'il est constitutif d'une pratique religieuse, voire d'un charisme (et d'une "performativité par écrans interposés", p. 177). Outre les stratégies de présentation et de mise en visibilité des pentecôtistes par le biais des technologies de la communication, l'étude de cas nous montre également l'émergence de la figure du télé-fidèle (" celui qui voit et participe au culte à distance ", p. 174) et des mutations à l'œuvre dans «l'action cérémonielle» (p. 174). Cette partie sur les « Modalités et enjeux de la présence en ligne », bien qu'interrogeant les pratiques religieuses, s'intéresse avant tout aux enjeux d'une «internationalisation» (Julliard), d'une expansion (Niculescu, Kirschleger) des pratiques par le biais des technologies de la communication (Mottier). 
6 La partie « Pratiques et rituels en ligne » comporte des questionnements très voisins, tout en insistant sur l'hybridation de pratiques en ligne et hors-ligne et sur l'intégration du numérique dans les pratiques quotidiennes. Des rencontres amoureuses confessionnelles (Lardellier), aux pratiques funéraires (Duteil-Ogata), en passant par la prière à distance et ses supports (Catellani, Douyère), la troisième partie est riche d'enseignements. Pascal Lardellier, dans « Rencontres religieuses virtuelles, très réelle endogamie... ", part du présupposé qu'une société multiculturelle laisserait la possibilité d'une plus grande hétérogamie confessionnelle. À travers une étude du terrain de rencontres confessionnelles en ligne (chrétiennes, juives et musulmanes), il montre que le web est un espace d'endogamie religieuse, à l'œuvre sur le marché en ligne des « sites culturels». Catellani, dans « Pastorale et prière en ligne : le cas du site Notre Dame du Web ", s'intéresse à la façon dont la prière peut " prendre corps » dans un environnement numérique. Dans une approche compréhensive et mobilisant la sémiotique, il tente d'« observer des formes d'énonciation, des types de construction de l'identité et des formes d'(auto)représentation des acteurs sociaux dans le texte" (p. 204) en ligne. Avec «Accompagner et susciter la prière à distance. Les prières méditatives en diaporamas de l'abbaye bénédictine Sainte-Marie des DeuxMontagnes ", David Douyère s'intéresse aussi à la prière et en particulier aux " supports de prière informatisés » comme autant de "ressources religieuses » pour la nouvelle évangélisation et produites par des moniales bénédictines du Québec. Sa recherche interroge alors les diaporamas - "dispositif de "prière assistée par ordinateur" (p. 217)»- comme supports de la communication de la foi chrétienne par l'image. Il montre également comment cette démarche d'aide à la «mise en relation avec le divin» (p. 218) s'inscrit dans une perspective plus large d'évangélisation de nature multimédia. Cette partie s'achève avec la proposition de Fabienne Duteil-ogata qui, dans «Nouvelles pratiques funéraires japonaises: de la tombe-ordinateur à la tombe-online ", expose un cas de transposition de pratiques funéraires traditionnelles japonaises hors-ligne à une technologisation grandissante de ces pratiques et l'usage d'une "tombe-online", devenu espace mémoriel. Cette pratique - dévolue aux monastères - consiste d'abord en une numérisation de l'identité du défunt et une sépulture ornée d'un écran. Dans un second temps, il s'agit d'une mise en accès des portraits du défunt sur Internet permettant à la famille de se recueillir n'importe où dans le monde. Duteil-Ogata montre, à travers plusieurs modèles de dispositifs funéraires, des formes d'individuation et d'esthétisation du défunt.

7 La dernière partie de l'ouvrage "Questions d'autorité » interroge les mutations à l'œuvre au sein des communautés religieuses. Se pose ici la question de l'autorité de la parole religieuse. Est-elle conservée par les institutions ? Comment ces dernières fontelles face à la libéralisation de la parole offerte par le média? Ainsi, Constance Arminjon, montre dans «La diffusion d'Internet et l'ouverture d'un nouveau champ dans le droit islamique chiite» que dans l'islam chiite, les "sources d'imitation» inventées au $\mathrm{xIX}^{\mathrm{e}}$ siècle se renouvellent au contact du média Internet pour la normalisation du droit chiite. La " dépersonnalisation » des relations des fidèles avec les autorités n'entraîne pas une dissolution de l'autorité, mais une recomposition du lien. Ainsi, "[les nouveaux médias] constituent désormais des objets de l'autorité normative des sources d'imitation chiites» (p. 250). Julien Bondaz, dans "Images cultuelles et écrans mourides, deux cas de controverse iconographique et religieuse sur Internet ", s'intéresse quant à lui à deux situations impliquant l'apparition de Cheikh mourides au Sénégal et sujets à controverse. Il montre ainsi « comment la discussion, le 
débat ou la polémique qui naissent à leur sujet transforment les internautes en experts techno-religieux, et plus généralement comment les usages religieux des nouvelles technologies participent à la modélisation des formes de croyance et des rapports aux images inédits» (p. 263). Chrystal Vanel, dans "Mormonisme et Internet entre promotion institutionnelle et régulation de l'utilisation individuelle ", nous explique que l'Église de Jésus-Christ-des-Saints-des-Derniers-Jours, par crainte d'une trop grande liberté de consultation d'Internet par les fidèles et dans le souci de conserver une autorité de la parole, impose un ensemble de règles d'usages du média à des fins prosélytes et de renforcement de la foi, mais aussi une régulation des accès de la famille à Internet. Laurence Podselver s'intéresse, dans « De la visibilité à la présence virtuelle. Quelques fragments de l'expression du judaïsme sur le Net ", à la présence numérique de Juifs Loubavitch qui, ayant intégré les pratiques numériques, vivent "dans » le numérique et non plus «avec ». Minoritaires, les Loubavitch, nous dit Podselver, multiplient les sites Internet entraînant ainsi une "surreprésentation» (p. 291) de leurs groupes. Pour clôturer cette partie - et l'ouvrage - l'article de Mia Lövheim, spécialiste de la recherche dans le champ d'Internet et religion, nous propose de nous pencher sur les blogs de jeunes femmes musulmanes scandinaves. Dans «Une voix à elles: jeunes, musulmanes et blogueuses", l'auteur montre qu'une expression minoritaire chez des jeunes femmes musulmanes peut être entendue comme une forme d'autonomisation dont le dispositif blog est l'instrument.

Bien que "novateur et pionnier ", l'ouvrage n'en est pas moins qu'un début. Les études futures gagneraient à rompre avec la posture proposée par certains contributeurs consistant à mettre en avant une "visée comparatiste » entre les catégories réel-virtuel et à entreprendre le web comme terrain au sens anthropologique et sociologique. Le caractère parfois désuet d'une terminologie numérique, comme celle du cyberespace, montre qu'un ancrage en sociologie et anthropologie classiques ne peut faire l'économie d'une plus grande prise en compte des travaux sur les cultures numériques en sciences de l'information et de la communication et en sociologie des médias. Les recherches futures s'emploieront, sans doute, à redéfinir certaines notions. Cet ouvrage collectif constitue un apport " pionnier » important dans l'analyse du fait religieux sur le web. Il comporte des articles tant de spécialistes reconnus dans le domaine, que de nouveaux venus sur le "terrain» du web et quantité d'articles de grande qualité scientifique qui, à n'en pas douter, intéresseront novices et spécialistes tant par la variété des approches que par celle des confessions et dénominations étudiées. 